\title{
EPR linewidth variation, spin relaxation times, and exchange in amorphous hydrogenated carbon
}

\author{
R. C. Barklie and M. Collins \\ Department of Physics, Trinity College, Dublin 2, Ireland \\ S. R. P. Silva \\ School of Electronic Engineering, Information Technology and Mathematics, \\ University of Surrey, Guilford, Surrey GU2 5XH, United Kingdom
}

(Received 12 August 1999)

\begin{abstract}
Electron paramagnetic resonance (EPR) measurements have been made of amorphous hydrogenated carbon $(a-\mathrm{C}: \mathrm{H})$ films grown by plasma enhanced chemical vapor deposition (PECVD) with negative self-bias voltages $V_{b}$ in the approximate range $10-540 \mathrm{~V}$. For $V_{b}<100 \mathrm{~V}$, as the film changes from polymerlike to diamondlike, the changes in linewidth and shape are interpreted in terms of changes to two contributions-one due to dipolar interactions between the unpaired spins and one due to unresolved lines arising from hyperfine interactions with $\mathrm{H}^{1}$. The former yields a Lorentzian line, the latter a Gaussian, and the resultant spectrum has the Voigt shape. The empirical relation $\Delta B_{\mathrm{pp}}^{\mathrm{G}}$ (in Gauss $)=(0.18 \pm 0.05) \times($ at. $\% \mathrm{H})$ between the peak-to-peak Gaussian contribution (in Gauss) $\Delta B_{\mathrm{pp}}^{\mathrm{G}}$ and the hydrogen content in atomic percentage is obtained. For $V_{b}>100 \mathrm{~V}$ the linewidth is shown to be dominated by the dipolar interactions and exchange and it decreases as $V_{b}$ increases; the change is shown to arise primarily from a change in the exchange interaction. Evidence for this comes from measurements which show that the spin-lattice relaxation time appreciably shortens and the spin-spin relaxation time lengthens as the bias voltage is increased. The magnitude and variation with bias of the linewidth are consistent with the EPR signal originating from the $\pi$-type radicals.
\end{abstract}

\section{INTRODUCTION}

Hydrogenated amorphous carbon, $a-\mathrm{C}: \mathrm{H}$, is a fascinating material because the possibility of varying the bonding configurations of the carbon atoms and also the hydrogen content allows it to be prepared with a wide range of characteristics. ${ }^{1,2}$ One method of preparing $a-\mathrm{C}: \mathrm{H}$ is plasmaenhanced chemical vapor deposition. The film properties are strongly affected by the incident ion energy which in turn is determined by the gas pressure and the negative self-bias voltage $V_{b}$ which develops between the live electrode and the glow space. ${ }^{3}$ At low bias voltages the films are "polymerlike" (PLC), soft, with wide optical gaps, a high photoluminescence efficiency and a high $(\approx 50 \%)$ hydrogen content. ${ }^{4}$ As the self-bias is increased the film initially becomes "diamondlike" (DLC), harder, with a smaller gap and a lower hydrogen content; with further increases in the bias the $s p^{2}$ content increases ${ }^{4}$ until eventually they become graphitelike (GLC). $a$-C:H with a particularly high $(\approx 80 \%)$ proportion of tetrahedrally bonded $s p^{3}$ carbon sites is often referred to as $t a-\mathrm{C}: \mathrm{H}$.

All the above forms of $a-\mathrm{C}: \mathrm{H}$ contain defects which are believed to affect the photoluminescence efficiency ${ }^{5}$ and the ability to dope the material. It is, therefore, of interest to know about these defects. One technique which has often been used to characterize those defects which are paramagnetic is electron paramagnetic resonance (EPR). In most cases a single resonance is reported which has a $g$ value of about 2.0028. ${ }^{6-8}$ However, it is still uncertain what these defects are and they could, for example, be either $s p^{2}$ clusters with an odd number of sites or single dangling bonds.
Changing the incident ion energy has little effect on the $g$ value but it does alter the linewidth. ${ }^{8-13}$ Several authors ${ }^{8-10}$ have reported that when the ion energy is increased from very low values there is an initial increase in the linewidth. One explanation proposed for this is that the spins are less able to tunnel to neighboring graphitic clusters as the energy is initially increased. ${ }^{8}$ At higher ion energies the linewidth decreases as the ion energy is further increased..$^{8-13}$ In this latter region the line is usually reported ${ }^{8-10,12,13}$ as having a Lorentzian shape although it has also been assumed to be Gaussian. ${ }^{11}$ In the latter case the change in width was attributed to a change in unresolved hydrogen hyperfine lines brought about by a change in the local $\mathrm{H} / \mathrm{C}$ ratio ${ }^{11}$ and it has been shown ${ }^{14}$ that changing the hydrogen content can affect the linewidth. On the other hand many authors ${ }^{8,12,13}$ have proposed that, on the basis that other mechanisms are inappropriate and that the line shape is Lorentzian, the narrowing is produced by exchange.

This work has two main aims-first, to find more evidence for the exchange narrowing process and second, to provide a comprehensive interpretation of the variation in linewidth as the $a-\mathrm{C}: \mathrm{H}$ is changed from polymerlike to diamondlike to graphitelike material. To try to achieve these aims we have made EPR measurements on a set of samples prepared by the plasma enhanced chemical vapor deposition (PECVD) method with a wide range of bias voltages from about 10 up to $540 \mathrm{~V}$. To look for evidence of the exchange interaction between the unpaired spins we have measured the spin-lattice, $T_{1}$, and spin-spin, $T_{2}$, relaxation times because it is known, for example, from the case of solid solutions of $\alpha$ - $\alpha$-diphenyl $\beta$-picryl hydrazyl (DPPH) in polystyrene, ${ }^{15}$ that increasing the strength of the exchange interaction has 
the effect of shortening $T_{1}$ and lengthening $T_{2}$. There have been a few measurements ${ }^{16}$ of $T_{1}$ and $T_{2}$ for different types of $a-\mathrm{C}: \mathrm{H}$ but this paper presents a systematic study of the effect on $T_{1}$ and $T_{2}$ of changing the bias voltage.

In Sec. II we briefly outline the sample preparation and EPR measurement procedures. Measurements of the linewidth, shape, $g$ value, and spin concentration are presented in Sec. III A. In Sec. III B we consider the analysis of the linewidth for samples grown with $V_{b}>100 \mathrm{~V}$, present the measurements of $T_{1}$ and $T_{2}$, and consider the evidence for exchange. The dependence of linewidth and shape on $V_{b}$ for $V_{b}<100 \mathrm{~V}$ is analyzed in Sec. III C; in this region the transformation from PLC to DLC occurs.

\section{EXPERIMENTAL DETAILS}

The $a-\mathrm{C}: \mathrm{H}$ samples were deposited using a $13.56 \mathrm{MHz}$ rf powered, capacitively coupled, PECVD system. All but one of the samples were deposited on silicon substrates placed on the rf driven electrode with feed gases of $\mathrm{CH}_{4}(10 \%)$ and $\mathrm{Ar}$ $(90 \%)$ at a constant pressure of 300 mTorr and with the rf power varied such that the dc self-bias voltage $V_{b}$ took values between -50 and $-540 \mathrm{~V}$ (from now on the minus sign will be omitted). The driven electrode was kept at room temperature by a closed-loop cooling system but the surface of the samples during deposition was at a slightly higher temperature. The atomic hydrogen content measured using elastic recoil detection analysis (ERDA) is 25 at. $\%$ at $V_{b}$ $=50 \mathrm{~V}, 13$ at. $\%$ at $100 \mathrm{~V}$ and thereafter probably decreases only slightly as $V_{b}$ is raised to $540 \mathrm{~V}$. The percentage $s p^{2}$ content was estimated to be $63,54,66,78,72,80,76,90$, and close to $100( \pm 10 \%)$ for $V_{b}$ values of $50,100,150,180$, $265,320,380,460$, and $540 \mathrm{~V}$, respectively. The $s p^{2}$ content was obtained by the method proposed by Berger et al. ${ }^{17}$ using a CM30 Philips microscope equipped with a dedicated Gatan model 666 electron energy-loss spectrometer and operated at $100 \mathrm{keV}$. Strictly speaking, the value obtained is for the $\pi$ content and, therefore, it gives the $s p^{2}$ percentage only if there is little or no $s p^{1}$ bonding within the $a-\mathrm{C}: \mathrm{H}$ matrix. This trend towards higher $s p^{2}$ content ( $\pi$ hybridized bonding) at higher ion energies is typical for such films. ${ }^{4,11}$ One sample was deposited on a silicon substrate placed on the earthed electrode at room temperature. The feed gases were $\mathrm{CH}_{4}(30 \mathrm{sccm})$ and $\mathrm{He}(75 \mathrm{sccm})(\mathrm{sccm}$ denotes cubic centimeter per minute at STP), the total pressure $200 \mathrm{mtorr}$ and the rf power $200 \mathrm{~W}$. For this arrangement the accelerating self-bias voltage is only about $10 \mathrm{~V}$. The hydrogen content was measured using ERDA to be about 50 at. \%, the $s p^{2}$ content $60 \pm 10 \%$, and the Tauc and $E_{04}$ gaps are 2.6 and 3.4 $\mathrm{eV}$, respectively. ${ }^{18}$ This film was removed from the substrate before the EPR measurements were made.

EPR measurements were made at room temperature using $100 \mathrm{kHz}$ field modulation, a microwave frequency of about 9.9 GHz, and a $\mathrm{TM}_{011}$ mode cavity. To avoid distortion of the spectrum the modulation amplitude was kept less than or equal to one third of the peak-to-peak linewidth. Each $g$ value was calculated by comparing the field position of the resonance with that of a resonance with known $g$ value-for example that of $\mathrm{F}^{+}$centers in $\mathrm{MgO}$ with $g=2.0023$. The field range was calibrated with a proton NMR probe which also gave absolute field values. Spin populations were found

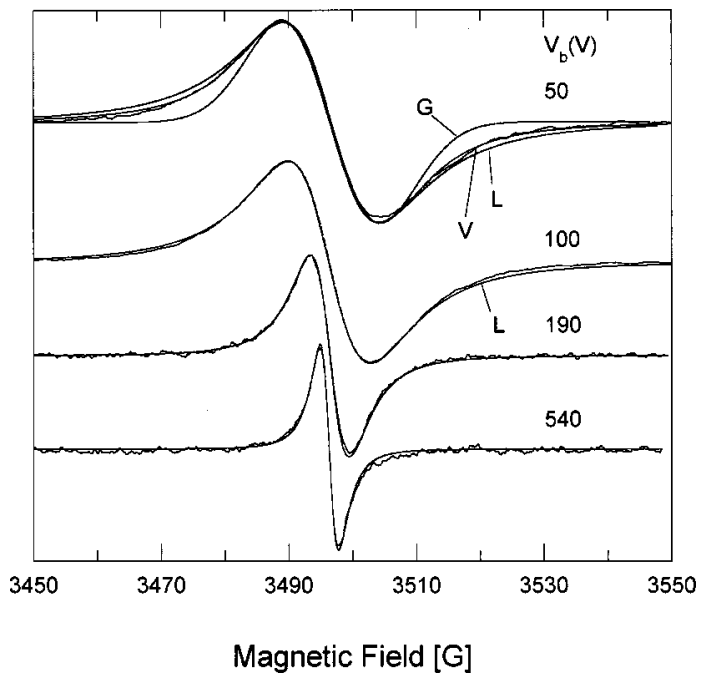

FIG. 1. EPR spectra showing the effect of changing the negative self-bias voltage from 50 to $540 \mathrm{~V}$. The smooth lines are computed fits. Only Lorentzian line fits are shown for $V_{b}=100,190$, and 540 V. For $V_{b}=50 \mathrm{~V}$, Lorentzian (L) and Gaussian (G) fits are shown but the best fit has a Voigt (V) line shape with parameters given in the text. Peak-to-peak heights have been normalized.

by comparing the integrated area of the signal with that of a standard Varian sample of pitch in $\mathrm{KCl}$; the absolute spin populations (and average spin densities) are estimated to be correct to within a factor of 2 , but their relative values are correct to within approximately $\pm 20 \%$.

\section{RESULTS, ANALYSIS, AND DISCUSSION}

\section{A. Line shape, width, and spin concentration-the results}

Figure 1 shows that one effect of increasing the negative self-bias voltage $V_{b}$ from 50 to $540 \mathrm{~V}$ is to narrow the EPR line. However, when $V_{b}$ is reduced from 50 to about $10 \mathrm{~V}$ (substrate on earthed electrode) the peak-to-peak linewidth, $\Delta B_{\mathrm{pp}}$, is decreased to $11.3 \pm 0.8 \mathrm{G}$. Figure 1 also shows that another effect of increasing $V_{b}$ is to change the line shape from one which is intermediate between a Gaussian and Lorentzian to a Lorentzian. One parameter which is indicative of the line shape is the ratio of the full width at half height, $\Delta B_{1 / 2}$, of the integrated spectrum to $\Delta B_{\mathrm{pp}}$; $\Delta B_{1 / 2} / \Delta B_{\mathrm{pp}}$ is 1.1776 for a Gaussian and 1.7321 for a Lorentzian. ${ }^{9}$ The values for the spectra are $1.42 \pm 0.15$, $1.48 \pm 0.09, \quad 1.57 \pm 0.12, \quad 1.63 \pm 0.11, \quad 1.69 \pm 0.11, \quad 1.72$ \pm 0.16 , and $1.80 \pm 0.19$ of samples deposited at $V_{b}=10,50$, $100,190,265,500$, and $540 \mathrm{~V}$ respectively. These values and the fits in Fig. 1 show that at $V_{b}=100 \mathrm{~V}$ the line shape is close to Lorentzian and that at $V_{b} \geqslant 190 \mathrm{~V}$ they are all Lorentzian within experimental error. Figure 1 shows that at $V_{b}=50 \mathrm{~V}$ the spectrum can be well fitted by a Voigt line ${ }^{20}$ which is a convolution of a Gaussian and a Lorentzian and is appropriate when these two contributions are independent. From the fit we obtain $\Delta B_{\mathrm{pp}}^{\mathrm{L}}=11.1 \pm 0.5 \mathrm{G}$ and $\Delta B_{\mathrm{pp}}^{\mathrm{G}}=8.2 \mp 0.5 \mathrm{G}$ for the above two components, respectively. The total linewidth of this fit is $15.5 \mathrm{G}$, which is the same as the experimentally determined width and, since the components are not both Lorentzian, is different from their sum. The spectrum at $V_{b}=100 \mathrm{~V}$, which is nearly but not 


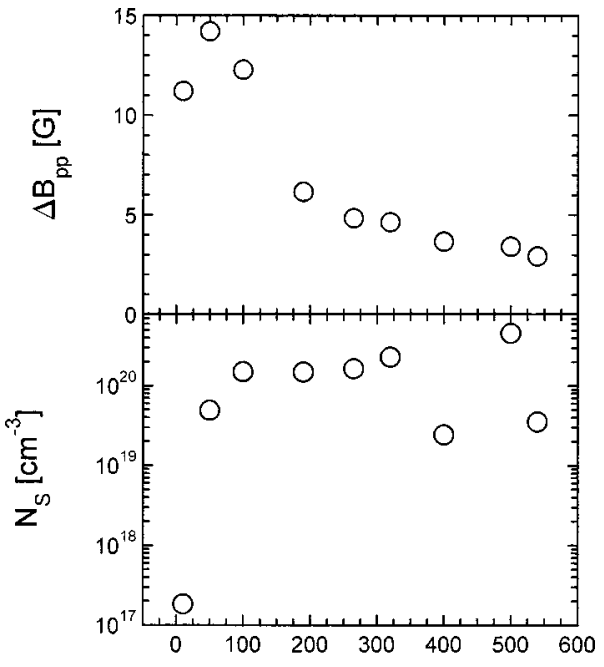

Negative self bias [V]

FIG. 2. The dependence on bias voltage of the peak-to-peak linewidth and spin concentration.

quite a Lorentzian, can be better fitted to a Voigt shape with $\Delta B_{\mathrm{pp}}^{\mathrm{L}}=11.7 \pm 0.5 \mathrm{G}$ and $\Delta B_{\mathrm{pp}}^{\mathrm{G}}=5.1 \mp 0.5 \mathrm{G}$. We have also tried fitting the spectra for $V_{b}=50$ and $100 \mathrm{~V}$ to superpositions of a single Gaussian and single Lorentzian line with the same $g$ value. These fits are as good as those with the Voigt shape; the parameters of the fits for the $V_{b}=50$ and $100 \mathrm{~V}$ spectra, respectively, are $\Delta B_{\mathrm{pp}}^{\mathrm{L}}=15.3 \mathrm{G}, \quad \Delta B_{\mathrm{pp}}^{\mathrm{G}}=3 \mathrm{G}$, $h_{\mathrm{pp}}^{\mathrm{L}} / h_{\mathrm{pp}}^{\mathrm{G}}=1.3$ and $\Delta B_{\mathrm{pp}}^{\mathrm{L}}=13.2 \mathrm{G}, \quad \Delta B_{\mathrm{pp}}^{\mathrm{G}}=15.8 \mathrm{G}, \quad h_{\mathrm{pp}}^{\mathrm{L}} / h_{\mathrm{pp}}^{\mathrm{G}}$ $=2.9$, where $h_{\mathrm{pp}}$ is the peak-to-peak height of the spectrum. Although the nature of the fit is uncertain there is no doubt that as $V_{b}$ is reduced from 100 to $50 \mathrm{~V}$ the line shape becomes less Lorentzian and more Gaussian in character. Because of the poor signal/noise ratio of the weak spectrum for $V_{b}=10 \mathrm{~V}$ we have not attempted to fit the spectrum but, as the ratio $\Delta B_{1 / 2} / \Delta B_{\mathrm{pp}}=1.42 \pm 0.15$ makes clear, it too is intermediate between a Gaussian and Lorentzian shape.

No dependence on bias voltage was observed in the $g$ value, which remains within \pm 0.0002 of 2.0025 as $V_{b}$ is increased from 10 to $540 \mathrm{~V}$, but Fig. 2 shows that the spin concentration $N_{s}$, as well as the linewidth, does depend on $V_{b}$. Several other authors ${ }^{8-11,21}$ have reported the large increase in $N_{s}$ from $\approx 10^{17} \mathrm{~cm}^{-3}$ up to $\approx 10^{20} \mathrm{~cm}^{-3}$ as the bias is increased from close to zero up to $\approx 100 \mathrm{~V}$. The increase has been attributed to an increase in disorder, ${ }^{1}$ a loss of hydrogen, ${ }^{1,11}$ an increase in strain, ${ }^{8}$ and an increase in the correlation energy $U .{ }^{9}$ Above $V_{b} \approx 100 \mathrm{~V}$ the spin concentration is less sensitive to changes in $V_{b}$ as noted, too, by others, ${ }^{8,9}$ although in the case of ta-C:H a drop from $2 \times 10^{20} \mathrm{~cm}^{-3}$ to $7 \times 10^{19} \mathrm{~cm}^{-3}$ was found ${ }^{13}$ as the energy per $\mathrm{C}$ atom increased from 100 to $270 \mathrm{eV}$ and Arcon et al. ${ }^{22}$ found $2.5 \times 10^{19} \mathrm{~g}^{-1}$ in $a-\mathrm{C}: \mathrm{H}$ deposited at $V_{b}=400 \mathrm{~V}$. The reason for the scatter in $N_{s}$ values in our samples for $V_{b}$ $\geqslant 300 \mathrm{~V}$ is not clear.

Along with the change in behavior of $N_{s}$ versus $V_{b}$ there is a change in the bias dependence of the linewidth. Several other authors ${ }^{8-10}$ also report that $\Delta B_{\mathrm{pp}}$ initially increases in $a-\mathrm{C}: \mathrm{H}$ as the bias increases from zero and many report that in $a-\mathrm{C}: \mathrm{H}$ (Refs. 8-11) as well as $t a-\mathrm{C}: \mathrm{H}$ (Ref. 13) and $t a-\mathrm{C}$ (Ref. 12) $\Delta B_{\mathrm{pp}}$ then decreases as the bias continues to in- crease. For $a-\mathrm{C}: \mathrm{H}$ the value of $V_{b}$ at which the maximum $\Delta B_{\mathrm{pp}}$ occurs depends slightly on the deposition system and conditions: Ristein et al. ${ }^{9}$ find the maximum at $V_{b}=90 \mathrm{~V}$ and, using a magnetically confined plasma, Silva et al. ${ }^{10}$ find it at $V_{b}=34 \mathrm{~V}$. It is well known that many of the other characteristics of $a-\mathrm{C}: \mathrm{H}$ change as the bias is increased. As has been pointed out ${ }^{4}$ three regions can be identified-a low bias region with a high $s p^{3}$ and hydrogen content giving soft "polymeric" films, a medium bias region with a lower hydrogen content but still quite a high $s p^{3}$ content giving harder "diamondlike" films, and a high bias region which gives more graphitelike films with a high $s p^{2}$ content. The transition from PLC to DLC is estimated to be essentially complete at $V_{b} \approx 100 \mathrm{~V} .^{9,23}$ It is clear, therefore, that the rapid increase in $N_{s}$ and increase in $\Delta B_{\mathrm{pp}}$ as $V_{b}$ increases mark the change from PLC to DLC, and that the next stage of little change in $N_{s}$ and a decrease in $\Delta B_{\mathrm{pp}}$ corresponds to the transition from DLC to GLC. It is convenient, therefore, to consider what determines the linewidth and its dependence on bias in two stages-first for $V_{b} \geqslant 100 \mathrm{~V}$ (DLC to GLC) and second for $V_{b}<100 \mathrm{~V}$ (PLC to DLC).

\section{B. Analysis of linewidth variation for $V_{b} \geqslant 100 \mathrm{~V}$ : Relaxation times and exchange}

Several authors ${ }^{6,8,12,13}$ have attributed the narrowing of the line, as bias voltage increases in the above range, to the exchange interaction between the spins. This attribution has been arrived at largely by excluding, at least as dominant contributions, other factors which might contribute to the linewidth. The width cannot be determined by a spread in $g$ values because $\Delta B_{\text {pp }}$ was found to be independent of the field at which the resonance was recorded, i.e., of microwave frequency ${ }^{14}$ and also the line shape would not then be Lorentzian. Nor can the decrease in linewidth be determined solely by a change in the dipolar interaction between the spins since, as Fig. 2 shows, the decrease is not always accompanied by a decrease in spin concentration and is sometimes accompanied by an increase in $N_{s} \cdot{ }^{10,11}$ Nor, in this bias region, can the linewidth be determined by unresolved hydrogen hyperfine interactions since, first, these lead to a line shape which is close to Gaussian ${ }^{24}$ rather than Lorentzian; second, as noted elsewhere, ${ }^{12}$ the linewidth of the resonance in $t a-\mathrm{C}$ varies with $s p^{2}$ fraction in a way very similar to that observed for $t a-\mathrm{C}: \mathrm{H}$; and third, the hydrogen content does not greatly decrease as the bias is increased. For example, Kleber et al. ${ }^{11}$ found that for $a-\mathrm{C}: \mathrm{H}$, increasing the ion energy from 30 to $170 \mathrm{eV}$ [corresponding approximately to a bias increase of 75 to $425 \mathrm{~V}$ (Ref. 4)] caused $\Delta B_{\mathrm{pp}}$ to decrease from 18 to $3 \mathrm{G}$ although the hydrogen content only dropped from 45 to 32 at. \%, and in $t a-\mathrm{C}: \mathrm{H}$ (Ref. 13) a drop in $\Delta B_{\mathrm{pp}}$ from 3.8 to $1.8 \mathrm{G}$ was only accompanied by a reduction of hydrogen content from 27 to 22 at. \%. This leaves exchange narrowing as a possible mechanism which is plausible for the following reasons. Whether the film is $a-\mathrm{C}: \mathrm{H}$, $t a-\mathrm{C}: \mathrm{H}$, or $t a-\mathrm{C}$, the decrease in $\Delta B_{\mathrm{pp}}$, in this higher bias region, is always accompanied by a decrease in the optical gap and an increase in the $s p^{2}$ fraction; ${ }^{8-13}$ a correlation of linewidth and gap has already been noted. ${ }^{25}$ Regarding the gap, Robertson and O'Reilly ${ }^{2}$ showed that the most stable arrangement of $s p^{2}$ sites is in compact clusters of fused six- 
fold rings and that the gap varies inversely as the cluster size. This model was later modified ${ }^{26}$ to account for the reduction in clustering produced by disorder and the presence of olefinic chains as well as aromatic rings. Olefinic chains could also of course delocalize the $\pi$ electrons and therefore act as a pseudocluster. Nevertheless increasing the $s p^{2}$ content is still expected to increase the cluster size and decrease the gap. As noted earlier, many authors ${ }^{6,8,12,13,25}$ have put forward the hypothesis that the Lorentzian line shape arises from exchange narrowing and that the reduction in the linewidth as the $s p^{2}$ content increases is associated with the greater delocalization of the unpaired electrons as the cluster size increases. It should be noted, however, that it is the increased overlap of the wave functions brought about by the greater delocalization rather than the greater delocalization itself which increases the strength of the exchange interaction. Because the spin-lattice, $T_{1}$, and spin-spin, $T_{2}$, relaxation times are sensitive to the existence of fluctuating fields produced by the motion of the spins which arises, in effect, from exchange or other causes, we have sought to test this hypothesis by measuring them as a function of bias voltage.

Homogeneously broadened lines have a Lorentzian shape and for them it is appropriate to use the Bloch equations which yield for $\Delta B_{\mathrm{pp}}$ and the peak-to-peak height $h_{\mathrm{pp}}$ of the signal the expressions ${ }^{27}$

$$
\Delta B_{\mathrm{pp}}=\left[\Delta B_{\mathrm{pp}}^{0}\right] S^{-1 / 2}=\left[2 /\left(3^{1 / 2} \gamma T_{2}\right)\right] S^{-1 / 2}
$$

and

$$
h_{\mathrm{pp}} / B_{1}=\left[\lim _{B_{1} \rightarrow 0}\left(h_{\mathrm{pp}} / B_{1}\right)\right] S^{3 / 2}
$$

where the saturation parameter $S=\left(1+\gamma^{2} B_{1}^{2} T_{1} T_{2}\right)^{-1}$ and $B_{1}$ is the amplitude of the rotating component of the microwave field at the sample; $\gamma$ is the gyromagnetic ratio.

We determined $T_{2}$ from $\Delta B_{\mathrm{pp}}^{0}$, the linewidth in the limit $S=1$, and $T_{1} T_{2}$ from a fit of Eq. (2) to the plot of $h_{\mathrm{pp}}$ versus $B_{1}$. The relevant value of $B_{1}$ is that at the sample. Since the introduction of the sample, in particular the Si substrate, can alter the cavity $Q$ and hence also $B_{1}$, it is necessary to calibrate $B_{1}$ versus the incident microwave power with the sample in place; this we did for each sample by also introducing a small piece of DPPH for which $T_{1}$ and $T_{2}$ are known. ${ }^{15}$

The values found for $T_{1}$ and $T_{2}$ are shown in Fig. 3. For completeness we have included values of $T_{1}, T_{2}$ for $V_{b}$ $=10$ and $50 \mathrm{~V}$. The spectra of these two samples are not Lorentzian and the Bloch equations do not apply but, nevertheless, following Abragam and Bleaney ${ }^{28}$ we loosely define a time $T_{2}$ by Eq. (1), and $T_{1} T_{2}$ is estimated as described above; although the actual values are uncertain it is clear that $T_{1}$ for the polymerlike sample $\left(V_{b}=10 \mathrm{~V}\right)$ is much longer than for the other samples. Since the spectrum for $V_{b}$ $=100 \mathrm{~V}$ is much closer to a Lorentzian, there is less uncertainty about the $T_{1} T_{2}$ value for this sample.

Figure 3 shows that as the bias is increased, $T_{1}$ decreases and $T_{2}$ increases. A similar trend is observed for the $T_{1}, T_{2}$ values of DPPH in polystyrene as the exchange interaction is increased by increasing the DPPH spin concentration. ${ }^{15}$ It is interesting to compare pairs of values of $T_{1}, T_{2}$ for $a-\mathrm{C}: \mathrm{H}$ with those of DPPH measured at $300 \mathrm{~K}$. Three of these pairs

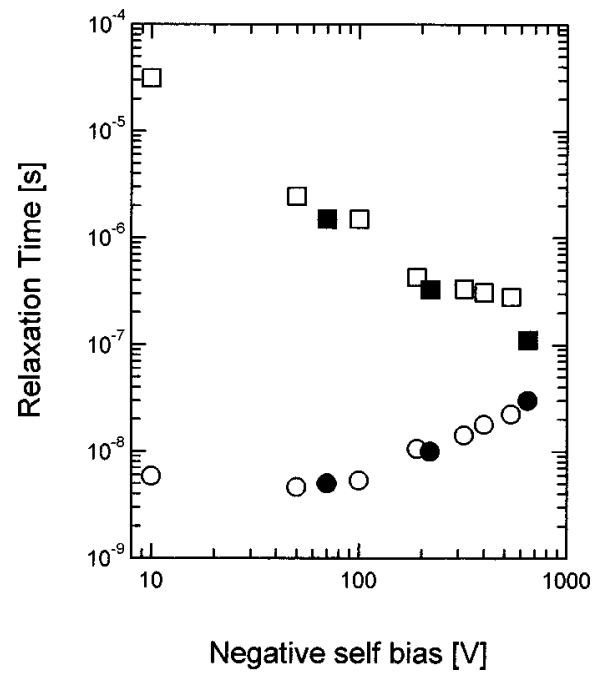

FIG. 3. The dependence on bias voltage of the spin-lattice, $T_{1}$, $(\square)$ and spin-spin, $T_{2}(\bigcirc)$ relaxation times of $a-\mathrm{C}: \mathrm{H}$. Also plotted are three pairs of $T_{1} T_{2}(\mathbf{\square}, \mathbf{0})$ values for DPPH in polystyrene, ${ }^{15}$ which in order of decreasing $T_{1}$ correspond to spin concentrations of $1 \times 10^{20}, 3 \times 10^{20}$, and $5 \times 10^{20} \mathrm{~cm}^{-3}$; they have been plotted such that the $T_{2}$ values match up with those of $a-\mathrm{C}: \mathrm{H}$.

for DPPH are shown in Fig. 3; the pairs correspond to DPPH spin concentrations of $1 \times 10^{20} \mathrm{~cm}^{-3}, 3 \times 10^{20} \mathrm{~cm}^{-3}$, and 5 $\times 10^{20} \mathrm{~cm}^{-3}$. They have been plotted such that the $T_{2}$ value for DPPH most closely matches its value for $a-\mathrm{C}: \mathrm{H}$. Given the uncertainties in $T_{1}$ it is remarkable how well the pairs match up. Furthermore the values for the earthed electrode sample are similar to the DPPH values of $T_{1}=1.3 \times 10^{-5} \mathrm{~s}$, $T_{2}=0.5 \times 10^{-8} \mathrm{~s}$ at the spin concentration of $10^{18} \mathrm{~cm}^{-3}$ below which both $T_{1}$ and $T_{2}$ become approximately independent of the spin concentration in DPPH. Our values for $\left(T_{1}, T_{2}\right)$ at $300 \mathrm{~K}$ agree reasonably well with the pairs of values $\left(<10^{-7} \mathrm{~s}, 1.8 \times 10^{-8} \mathrm{~s}\right),\left(2 \times 10^{-7} \mathrm{~s}, 2.5 \times 10^{-8} \mathrm{~s}\right)$, and $\left(2 \times 10^{-4} \mathrm{~s}, 0.4 \times 10^{-8} \mathrm{~s}\right)$ for what was labeled $\mathrm{ta}-\mathrm{C}$, diamondlike and polymerlike carbon, respectively; ${ }^{16}$ we have evaluated $T_{2}$ from the values of $\Delta B_{\mathrm{pp}}$ at $300 \mathrm{~K}$. There is not, however, a single pair of values of $\left(T_{1}, T_{2}\right)$ for a given type of $a-\mathrm{C}: \mathrm{H}$ as both the $s p^{2}$ content and energy gap can vary within any type. It is interesting to note that the $T_{1}$ values of carbon radicals in dextrose were found to decrease as the pyrolytic temperature was raised from 250 to $450{ }^{\circ} \mathrm{C} .{ }^{29} \mathrm{We}$ suggest that this occurs for the same reason that $T_{1}$ decreases with increasing $V_{b}$ for $a-\mathrm{C}: \mathrm{H}$, namely, that there is an increase in the $s p^{2}$ content leading to greater spin delocalization and a stronger exchange interaction.

To further analyze our results we make use of the densitymatrix method of Redfield which is described by Slichter. ${ }^{30}$ It is supposed that the spins experience a fluctuating field with components $B_{x}, B_{y}$, and $B_{z}$, in addition to the static field $B_{0}(/ / z)$. Such fluctuations may arise from variations in the dipolar or hyperfine field produced by the motion of the spins arising, in effect, from exchange or, for example, from hopping. $B_{z}$ will affect $T_{2}$ but not $T_{1}$. However, contributions to $B_{x}$ and $B_{y}$ at the Larmor frequency $\omega_{0}$ will affect $T_{1}$ and $T_{2}$. Assuming a simple exponential correlation function gives $^{30}$ 


$$
\frac{1}{T_{1}}=\gamma^{2}\left(\overline{B_{x}^{2}}+\overline{B_{y}^{2}}\right) \frac{\tau_{c}}{1+\omega_{0}^{2} \tau_{c}^{2}}, \quad \frac{1}{T_{2}}=\gamma^{2} \overline{B_{z}^{2}} \tau_{c}+\frac{1}{2 T_{1}},
$$

where $\tau_{c}$ is the correlation time of the fluctuation; the bar indicates the average value. Assuming further that $\overline{B_{x}^{2}}=\overline{B_{y}^{2}}$ $=\overline{B_{z}^{2}}=\overline{B^{2}}$ then

$$
\frac{1}{T_{1}}=\frac{2 \gamma^{2} \overline{B^{2}} \tau_{c}}{1+\omega_{0}^{2} \tau_{c}^{2}}, \quad \text { and } \quad \frac{1}{T_{2}}=\gamma^{2} \overline{B^{2}} \tau_{c}+\frac{\gamma^{2} \overline{B^{2}} \tau_{c}}{1+\omega_{0}^{2} \tau_{c}^{2}} .
$$

Equation (4) implies that as $\tau_{c}$ is decreased $T_{1}$ will decrease, will reach a minimum value of $\omega_{0} / \omega_{p}^{2}$ at $\tau_{c}^{-1} \equiv \omega_{c}$ $=\omega_{0}$ and then increase again and that $T_{2}$ will continuously increase and become equal to $T_{1}$ for $\omega_{0} \tau_{c} \ll 1$; we define $\omega_{p}^{2}=\gamma^{2} \overline{B^{2}}$. In the region $\omega_{p}<\omega_{c}<\omega_{0}$ Eq. (4) gives

$$
\frac{1}{T_{1}} \approx \frac{2 \omega_{c} \omega_{p}^{2}}{\omega_{0}^{2}} \text { and } \frac{1}{T_{2}} \approx \frac{\omega_{p}^{2}}{\omega_{0}},
$$

so that

$$
\omega_{c} \approx \omega_{0}\left(T_{2} / 2 T_{1}\right)^{1 / 2} .
$$

Since, for a Lorentzian line, the half-width at half height $\frac{1}{2} \Delta B_{1 / 2}=1 / \gamma T_{2}$, then Eq. (5) implies that

$$
\frac{1}{2} \gamma \Delta B_{1 / 2}=\omega_{p}^{2} / \omega_{c} .
$$

This is the expression given by Anderson and Weiss ${ }^{31}$ for an exchange narrowed line where $\omega_{c}$ represents the exchange frequency $\omega_{e}$.

In the slow motion limit when $\tau_{c} \geqslant T_{2}$ the model breaks down and the linewidth is given by the static field distribution over the spin sites together with any contribution from the spread in $g$ values. In the case of dipolar broadening in magnetically diluted substances, which is appropriate in $a-\mathrm{C}: \mathrm{H}$ with typically less than one unpaired spin per $100 \mathrm{C}$ atoms, the statistical theory ${ }^{32}$ predicts that the line shape is Lorentzian with

$$
\frac{1}{2} \Delta B_{1 / 2}=\frac{2 \pi^{2}}{3 \sqrt{3}} g \mu_{B} N_{s},
$$

where $N_{s}$ is the spin concentration in $\mathrm{cm}^{-3}$ and the field units are Gauss. Taking $g=2.0025$ and taking $\Delta B_{\mathrm{pp}}=\Delta B_{1 / 2} / \sqrt{3}$ for a Lorentzian gives

$$
\Delta B_{\mathrm{PP}}=8.12 \times 10^{-20} N_{s} .
$$

We now consider how the observed linewidths can be understood in terms of the above ideas. We will first consider the situation for $V_{b} \geqslant 100 \mathrm{~V}$. At $V_{b}=100 \mathrm{~V}$ the line shape is close to Lorentzian and the measured values are $\Delta B_{\mathrm{pp}}$ $=12.2 \pm 0.6 \mathrm{G}$ and $N_{s}=1.5 \times 10^{20} \mathrm{~cm}^{-3}$. Equation (9) predicts that under these conditions the dipolar interaction alone would yield a Lorentzian line with $\Delta B_{\mathrm{pp}}=12.2 \mathrm{G}$. The agreement is fortuitously good but clearly no other factor is required to explain the width and shape. This is analogous to the situation in DPPH for $N_{s} \leqslant 1 \times 10^{20} \mathrm{~cm}^{-3}$ when $\Delta B_{\mathrm{pp}}$ is no longer influenced by exchange. At $V_{b}=190 \mathrm{~V}$ the shape is Lorentzian within error, $N_{s}$ is unchanged but $\Delta B_{\mathrm{pp}}$ has dropped to $6.2 \mathrm{G}$. The $T_{1}, T_{2}$ values have changed to ones close to those for a DPPH spin concentration of $3 \times 10^{20} \mathrm{~cm}^{-3}$. The changes for DPPH are attributed to an increase in $\omega_{e}$ as the greater proximity of the spins at the higher concentration leads to increased wave function overlap. ${ }^{15}$ For $a-\mathrm{C}: \mathrm{H}$ we also attribute the changes to an increase in exchange interaction but in this case due to the increased delocalization of the spin wave functions associated with the large increase in the $s p^{2}$ content from $54 \%$ to nearly $80 \%$. It is tempting to use Eq. (6) and the ratio of $T_{2} / T_{1}$ to evaluate $\omega_{c}$ and hence also $\left(\overline{B^{2}}\right)^{1 / 2}$; for $V_{b}$ $=190 \mathrm{~V}$ we get $\omega_{c}=7 \times 10^{9} \mathrm{rad} \mathrm{s}^{-1}$ and $\left(\overline{B^{2}}\right)^{1 / 2}=46 \mathrm{G}$ (which satisfies $\omega_{p}<\omega_{c}<\omega_{0}$ ). However, these values should be treated with caution. When we apply the same procedure to DPPH with $N_{s}=3 \times 10^{20} \mathrm{~cm}^{-3}$ we get $\omega_{e}$ $=7.8 \times 10^{9} \mathrm{rad} \mathrm{s}^{-1}$ and $\left(\overline{B^{2}}\right)^{1 / 2}=50 \mathrm{G}$. As has been pointed out $^{31}$ the half-width at half height, $\frac{1}{2} \Delta B_{1 / 2}$, should be about equal to $\left(\overline{B^{2}}\right)^{1 / 2}$ in the absence of exchange. Equation (8) implies that at $N_{s}=3 \times 20 \mathrm{~cm}^{-3} \frac{1}{2} \Delta B_{1 / 2}$ $=21 \mathrm{G}$ so that the agreement is not very good.

As $V_{b}$ is increased further the width continues to decrease until at $540 \mathrm{~V}$ the width is close to that for DPPH at $N_{s}=5 \times 10^{20} \mathrm{~cm}^{-3}$. Here we get for DPPH $\omega_{e}$ $=2.7 \times 10^{10} \mathrm{rad} \mathrm{s}^{-1}$ and $\left(\overline{B^{2}}\right)^{1 / 2}=50 \mathrm{G}$; the latter does agree better with the value of $\frac{1}{2} \Delta B_{1 / 2}=35 \mathrm{G}$ evaluated from Eq. (8). At $V_{b}=540 \mathrm{~V}$ the values are $\omega_{c}=1.2 \times 10^{10} \mathrm{rad} \mathrm{s}^{-1}$ and $\left(\overline{B^{2}}\right)^{1 / 2}=42 \mathrm{G}$. The latter value so exceeds the $\frac{1}{2} \Delta B_{1 / 2}$ $=2.5 \mathrm{G}$ expected for $N=3.5 \times 10^{19} \mathrm{~cm}^{-3}$ that it suggests that, at least for $V_{b} \geqslant 400 \mathrm{~V}$, the spins have clustered. A local concentration of about $6 \times 10^{20} \mathrm{~cm}^{-3}$ is required to give $\left(\overline{B^{2}}\right)^{1 / 2}=42 \mathrm{G}$. Assuming that for $a-\mathrm{C}: \mathrm{H} \omega_{c}$ also represents $\omega_{e}$ and that $\omega_{e} \approx J / \hbar$, where $J$ is the exchange constant, then the value of $\omega_{e}=1.2 \times 10^{10} \mathrm{rad} \mathrm{s}^{-1}$ gives $J / k \approx 0.1 \mathrm{~K}$. Interestingly, evidence of clustering of spins in samples prepared under similar conditions has already been reported. ${ }^{22}$

We have seen that the changes in $\left(T_{1}, T_{2}\right)$ for $V_{b}$ $\geqslant 100 \mathrm{~V}$ can be accounted for by motional effects and we have assumed that, as in DPPH, these arise from exchange. It is known, however, that below about $300 \mathrm{~K}$ conduction in ion-beam sputtered $a$-C arises from variable range hopping ${ }^{33-35}$ and probably in ta-C:H also, ${ }^{35}$ so what justification is there for assuming that the "motion" is effectively produced by exchange rather than by hopping? Typically the conductivity falls by more than three orders of magnitude, ${ }^{33-35}$ reflecting the change in probability of an energy-upward hop, as the temperature drops from 300 to 40 $\mathrm{K}$. If linewidth narrowing is associated with hopping, a significant dependence of linewidth on temperature would be expected, but over the above temperature range the linewidth has been found to change by $\leqslant 20 \%$ in $t a-C$, diamondlike, graphitelike, and polymerlike carbon. ${ }^{16}$ On the other hand, this is consistent with exchange narrowing since $\omega_{e}$ should depend primarily on wave function overlap and hence be relatively insensitive to changes in temperature.

In summary, we explain the magnitude of the linewidth and its dependence on bias voltage in the range $V_{b} \geqslant 100 \mathrm{~V}$ in terms of a combination of dipolar broadening and exchange narrowing. At $V_{b} \approx 100 \mathrm{~V}$ the exchange is too slow to affect the linewidth which primarily is determined by dipolar interactions between the spins; the exchange may however be strong enough to shorten $T_{1}$, as is the case for DPPH. ${ }^{15}$ As 
TABLE I. EPR parameters, bias voltage, hydrogen content, and optical gap of $a$-C:H films deposited at low ion energies. $\Delta B_{\mathrm{pp}}^{T}$ is the measured peak-to-peak linewidth, $\Delta B_{\mathrm{pp}}^{\mathrm{L}}$ is calculated using the $N_{s}$ value and Eq. (9), and $\Delta B_{\mathrm{pp}}^{\mathrm{G}}$ is calculated using the above values of $\Delta B_{\mathrm{pp}}^{T}, \Delta B_{\mathrm{pp}}^{\mathrm{L}}$, and Eq. (12).

\begin{tabular}{|c|c|c|c|c|c|c|c|c|}
\hline \multicolumn{9}{|c|}{ Peak to Peak widths $(G)$} \\
\hline Max/Min & $\Delta B_{\mathrm{pp}}^{T}$ & $\Delta B_{\mathrm{pp}}^{\mathrm{L}}$ & $\Delta B_{\mathrm{pp}}^{\mathrm{G}}$ & $N_{s}\left(\mathrm{~cm}^{-3}\right)$ & $V_{b}(\mathrm{~V})$ & $\mathrm{H}($ at $\%)$ & $\mathrm{Gap}(\mathrm{eV})$ & Ref. \\
\hline Max? & $20.3(C)^{\mathrm{a}}$ & 16.2 & 9.1 & $2 \times 10^{20}$ & & & $2.0\left(E_{03}\right)$ & 8 \\
\hline Max & $17.2(A)^{\mathrm{a}}$ & 5.7 & 14.0 & $7 \times 10^{19}$ & & & $1.88\left(E_{03}\right)$ & \\
\hline Min & $8.7(A)^{\mathrm{a}}$ & 0.3 & 8.5 & $4 \times 10^{18}$ & & & $2.22\left(E_{03}\right)$ & \\
\hline $\operatorname{Max}$ & 14.6 & 10.5 & 7.7 & $1.3 \times 10^{20}$ & 90 & & $2.45\left(E_{04}\right)$ & 9 \\
\hline Min & 8.2 & 0.06 & 8.2 & $7.3 \times 10^{17}$ & 0 & & $4.00\left(E_{04}\right)$ & \\
\hline Max & $12 \pm 1$ & 16.2 & 0 & $2 \times 10^{20}$ & 34 & 24 & $3.05\left(E_{04}\right)$ & 10 \\
\hline Min & $3.2 \pm 1$ & 0.5 & 3.0 & $6 \times 10^{18}$ & 12 & 24 & $3.55\left(E_{04}\right)$ & \\
\hline Max? & 18 & 1.3 & 17 & $1.6 \times 10^{19}$ & $75^{\mathrm{b}}$ & 45 & $1.9\left(E_{\text {Tauc }}\right)$ & 11 \\
\hline Min? & 8 & 0.01 & 8.0 & $1.5 \times 10^{17}$ & & $45-50$ & $3.3\left(E_{04}\right)$ & 38 \\
\hline Max & $15.4 \pm 0.6$ & 4.0 & 13.2 & $4.9 \times 10^{19}$ & 50 & 25 & & Current \\
\hline Min & $11.3 \pm 0.8$ & 0.02 & 11.3 & $3 \times 10^{17}$ & 10 & 50 & $3.4\left(E_{04}\right)$ & Work \\
\hline
\end{tabular}

The films are grown on the cathode $(C)$ or the anode $(A)$.

${ }^{\mathrm{b}}$ The $V_{b}$ value is taken to be $75 \mathrm{~V}$ on the assumption (Ref. 4), that the ion energy of $30 \mathrm{eV}$ is $40 \%$ of $V_{b}$.

$V_{b}$ is raised above $100 \mathrm{~V}$ the increase in $s p^{2}$ content leads to an increasing delocalization of the spin wave function, and hence to an exchange interaction sufficiently large to produce an increasingly strong exchange narrowing. In this region the linewidth is correlated to the optical gap. We now consider the factors which determine the linewidth and its dependence on $V_{b}$ for $V_{b}<100 \mathrm{~V}$.

\section{Analysis of linewidth variation for $V_{b}<100 \mathrm{~V}$ : Dipolar and hyperfine interactions}

When $V_{b}$ is decreased below about $100 \mathrm{~V}$ the linewidth reaches a maximum and then decreases and the spin concentration falls rapidly; this is shown in Fig. 2 and has been observed by others. ${ }^{8,10}$ In addition we find that, as noted in Sec. III A, the line shape deviates from Lorentzian and becomes a mixture of Lorentzian and Gaussian. Table I summarizes the values of $\Delta B_{\mathrm{pp}}, N_{s}$, and other parameters at both the maximum $\Delta B_{\mathrm{pp}}$ and lowest $\Delta B_{\mathrm{pp}}$ (at the lowest ion energy used). The lowest ion energy used by Kleber et al. ${ }^{11}$ was $30 \mathrm{eV}$ [which corresponds to about $40 \%$ of $V_{b}$ (Ref. 4)] at which $\Delta B_{\mathrm{pp}}$ was probably close to its maximum value but had not begun to decrease. Similarly, for the cathode-type films of Schütte et al., ${ }^{8}$ the value of $\Delta B_{\mathrm{pp}}=20.3 \mathrm{G}$ is the largest observed but these films were not deposited at lower ion energies; on the other hand a turning point in $\Delta B_{\mathrm{pp}}$ was found for their anodelike films.

Table I shows the values of the dipolar contribution to the linewidth expected on the basis of the measured $N_{s}$ values and Eq. (9). It will be recalled that this fully accounted for the linewidth at $V_{b}=100 \mathrm{~V}$ but the values in the table indicate that the minimum values $\Delta B_{\mathrm{pp}}$, at the lowest ion energies, greatly exceed the expected dipolar contributions. The question, therefore, is not why does the linewidth decrease, but why does it not decrease much more? We suggest that the answer is that the unresolved hydrogen hyperfine interactions make an increasingly large contribution as the ion energy is reduced. $a-\mathrm{C}: \mathrm{H}$ deposited by the usual PECVD process has a hydrogen content which increases as the bias voltage is decreased ${ }^{11,36}$ and the increase is most rapid for
$V_{b}<100 \mathrm{~V} .{ }^{36}$ For our samples it is estimated to increase from 13 at. $\%$ at $100 \mathrm{~V}$ to about $50 \%$, typical of polymerlike $a-\mathrm{C}: \mathrm{H}$, at about $10 \mathrm{~V}$. Unresolved hyperfine lines are expected to give rise to a Gaussian line shape with an overall full width at half height given by ${ }^{24}$

$$
\left(\Delta B_{1 / 2}\right)^{2}=\left(8 \ln 2 / g^{2} \mu_{B}^{2}\right) \sum_{i, \iota} N_{\iota} \xi_{i} A_{i, \iota}^{2}\left[I_{i, \iota}\left(I_{i, \iota}+1\right)\right] / 3
$$

where $N_{\iota}$ is the number of nuclei in the $\iota$ th shell, $\xi_{i}$ is the relative abundance of the $i$ th isotope, and $A_{i, \iota}$ is the hyperfine interaction with the nuclei $i$ in the $\iota$ th shell. For the case of $H^{1}$, using $\Delta B_{1 / 2}=(2 \ln 2)^{1 / 2} \Delta B_{\mathrm{pp}}$ for a Gaussian, this becomes

$$
\left(\Delta B_{\mathrm{PP}}\right)^{2}=\sum_{\iota} N_{\iota} A_{\iota}^{2}
$$

where $A_{\iota}$ is here expressed in units of magnetic field. We therefore propose that the overall line shape and width for $V_{b}<100 \mathrm{~V}$ are determined by a combination of dipolar interaction and unresolved $\mathrm{H}^{1}$ hyperfine lines and, since these contributions should be independent, then the overall spectrum should have a Voigt profile with a total peak-to-peak width, $\Delta B_{\mathrm{pp}}^{T}$ given by ${ }^{37}$

$$
\Delta B_{\mathrm{PP}}^{T}=\frac{\Delta B_{\mathrm{PP}}^{\mathrm{G}}+0.9085 \Delta B_{\mathrm{PP}}^{\mathrm{G}} \Delta B_{\mathrm{PP}}^{\mathrm{L}}+0.4621\left(\Delta B_{\mathrm{PP}}^{\mathrm{L}}\right)^{2}}{\Delta B_{\mathrm{PP}}^{\mathrm{G}}+0.4621 \Delta B_{\mathrm{PP}}^{\mathrm{L}}},
$$

where $\Delta B_{\mathrm{pp}}^{\mathrm{G}}, \Delta B_{\mathrm{pp}}^{\mathrm{L}}$ are the peak-to-peak widths of the Gaussian and Lorentzian contributions which here are given by Eqs. (11) and (9), respectively. Using Eq. (12) and each value of $\Delta B_{\mathrm{pp}}^{\mathrm{L}}$, given in Table I, we can calculate the value required for $\Delta B_{\mathrm{pp}}^{\mathrm{G}}$ so that $\Delta B_{\mathrm{pp}}^{T}$ is equal to that of the recorded spectrum; these $\Delta B_{\mathrm{pp}}^{\mathrm{G}}$ values are also listed in Table I. We now need to consider the evidence for and against this proposed explanation of the linewidth and shape. 
Firstly, the model implies that as $V_{b}$ falls below about 100 $\mathrm{V}$, such that $N_{s}$ falls and $\mathrm{H}$ at. \% rises more rapidly, the line shape should change from Lorentzian to a Voigt profile. The change in shape we observe is consistent with this proposal and Sadki et al. ${ }^{38}$ also find that the line shape of a polymerlike sample, with $45-50$ at. $\% \mathrm{H}, E_{04}=3.3 \mathrm{eV}, N_{s}=1.5$ $\times 10^{17} \mathrm{~cm}^{-3}$, and $\Delta B_{\mathrm{pp}}=8 \mathrm{G}$, has both a Lorentzian and Gaussian component; by contrast their diamondlike sample with 25 at. $\% \mathrm{H}, E_{04}=1.3 \mathrm{eV}, N_{s}=4.5 \times 10^{20} \mathrm{~cm}^{-3}, \Delta B_{\mathrm{pp}}$ $=4 \mathrm{G}$ has a Lorentzian shape (and, we suggest, is exchange narrowed). Unfortunately, as noted previously, we cannot determine from the goodness of fit whether the line shape at $V_{b}=50 \mathrm{~V}$ is a Voigt profile or a superposition of one Lorentzian and one Gaussian line. Ristein et al. ${ }^{9}$ state, however, that for $V_{b} \geqslant 50 \mathrm{~V}$ the shape is Lorentzian but did not determine the shape at $V_{b}<50 \mathrm{~V}$. Silva et al. ${ }^{10}$ and Schütte et al. ${ }^{8}$ claim that the line shape for their samples at all bias values is Lorentzian. Therefore, although there is general agreement that the line shape is Lorentzian at the higher bias values the evidence is contradictory for the lower bias values and there is a need for further careful measurements.

Second, the model implies that for $N_{s}<10^{19} \mathrm{~cm}^{-3}$ the dipolar contribution to $\Delta B_{\mathrm{pp}}$ drops below $1 \mathrm{G}$ and therefore the linewidth is dominated by the unresolved $\mathrm{H}^{1}$ hyperfine lines. Considering, first, the minimum $\Delta B_{\mathrm{pp}}$ values (Table I) we see that, with one exception, the estimated $\Delta B_{\mathrm{pp}}^{\mathrm{G}}$ values lie in the approximate range $8-11 \mathrm{G}$. The exception is the value $\Delta B_{\mathrm{pp}}^{\mathrm{G}}=3 \mathrm{G}$ obtained by Silva et al. ${ }^{10}$ this is interesting because the $\mathrm{H}$ at. $\%$ is exceptionally low in this case-only $24 \%$ - and Eq. (11) implies that $\Delta B_{\mathrm{pp}}^{\mathrm{G}}$ would also be expected to be low. We now consider whether $\Delta B_{\mathrm{pp}}^{\mathrm{G}}$ $=8-11 \mathrm{G}$ is plausible for a $\mathrm{C}: \mathrm{H}$ ratio of about $1: 1$. One comparison to make is with the EPR spectrum of cis-rich polyacetylene $(\mathrm{CH})_{n}$ which is a single line, near $g=2$, which is nearly Gaussian with $\Delta B_{\mathrm{pp}}=8.5 \mathrm{G}$ at $295 \mathrm{~K}$ and $11 \mathrm{G}$ at $82 \mathrm{~K}^{39}$ Unlike the trans- $(\mathrm{CH})_{n}$ which has a Lorentzian, motionally narrowed line, the cis spectrum is well fitted to an immobile, delocalized soliton which is computed to have a spectrum of Gaussian shape with $\Delta B_{\mathrm{pp}}=12 \mathrm{G}$ due to the unresolved $\mathrm{H}^{1}$ hyperfine lines. ${ }^{39}$ Another comparison to make is with the $\Delta B_{\mathrm{pp}}$ values computed using Eq. (11) and the isotropic hydrogen hyperfine splittings for the benzene, naphthalene, and anthracene anions ${ }^{40}$ which we calculate are $9.2,10.5$, and $10.1 \mathrm{G}$, respectively. Of course this ignores contributions from the anisotropic terms but inclusion of these, even if known, would probably not much alter the values. The above values are reasonable being all about half the "extent," that is the spacing of the outermost lines, of the above spectra which is 22.5, 27.3, and $27.6 \mathrm{G}$, respectively. ${ }^{40}$ It is worth noting that in these cases the change in the degree of localization has little effect on the "extent" of the spectrum because the anions are isolated from each other in solution and so there is no wave function overlap.

Unlike the $\pi$-type organic radicals described above, the $\sigma$-type radicals have larger hyperfine interactions. Calculating $\Delta B_{\mathrm{pp}}$ as above yields about $70 \mathrm{G}$ for the alkyl radical $-\mathrm{CH}_{2}-\mathrm{CH}-\mathrm{CH}_{2}-$ and $50 \mathrm{G}$ for the mixed $\pi / \sigma$ allyl radical $-\mathrm{CH}_{2}-\mathrm{CH}-\mathrm{CH}=\mathrm{CH}-\mathrm{CH}_{2}-$. We conclude, therefore, that $\Delta B_{\mathrm{pp}}^{\mathrm{G}}=8-11 \mathrm{G}$ is compatible with what would be expected from unresolved $\mathrm{H}^{1}$ hyperfine interactions for $\pi$-type conjugated radicals with 50 at. $\% \mathrm{H}$; it is incompatible with that expected for $\sigma$-type radicals such as the alkyl radical.

As shown earlier, for the PLC samples, it is possible to estimate $\Delta B_{\mathrm{pp}}^{\mathrm{G}}$ if the spin concentration is known and the spectrum has a Voigt line shape. It would be useful to have a relation between $\Delta B_{\mathrm{pp}}^{\mathrm{G}}$ and the hydrogen content from which the latter could then be evaluated. Equation (11) suggests that $\Delta B_{\mathrm{pp}}^{\mathrm{G}}$ might be proportional to (at. $\left.\% \mathrm{H}\right)^{1 / 2}$ but changing the hydrogen content is also likely to change the hyperfine terms $A_{\iota}$. There is also likely to be a distribution of configurations - that is, of $N_{\iota}$ and $A_{\iota}$ values. We therefore put forward an empirical relationship

$$
\Delta B_{\mathrm{PP}}^{\mathrm{G}}(\mathrm{G})=(0.18 \pm 0.05) \times(\text { at. } \% \mathrm{H}) .
$$

The value of $0.18 \pm 0.05$ is just the mean of the values $\Delta B_{\mathrm{pp}}^{\mathrm{G}} /($ at. $\% \mathrm{H})$, given in Table I, of $3.2 \mathrm{G}$ at $24 \%, 8 \mathrm{G}$ at $45-50 \%$, and $11.3 \mathrm{G}$ at $50 \%$. For the other minimum linewidth values of 8.7 and $8.2 \mathrm{G}$ with $\Delta B_{\mathrm{pp}}^{\mathrm{G}}$ estimated to be 8.5 and $8.2 \mathrm{G}$, the empirical relation implies an atomic $\mathrm{H} \%$ of just under $50 \%$ which is reasonable considering the method and conditions used in preparing the samples.

Table I shows, however, that for the maximum linewidths the values estimated for $\Delta B_{\mathrm{pp}}^{\mathrm{G}}$ are rather high-higher in some cases than for samples with the minimum linewidths which, being deposited at lower biases, should have a higher hydrogen content. Also, in the case of our sample deposited at $V_{b}=50 \mathrm{~V}$, the values of $\Delta B_{\mathrm{pp}}^{\mathrm{L}}$ and $\Delta B_{\mathrm{pp}}^{\mathrm{G}}$ evaluated using Eqs. (9) and (12) are less than and greater than, respectively, the values obtained from the Voigt fit. The values found for $\Delta B_{\mathrm{pp}}^{\mathrm{G}}$ will be too high if those calculated for $\Delta B_{\mathrm{pp}}^{\mathrm{L}}$ [using Eq. (9)] are too low as will happen if the $N_{s}$ values given in Table I are too low. In the case of those samples with $N_{s}$ $>10^{20} \mathrm{~cm}^{-3}$ the increase required to give plausible $\Delta B_{\mathrm{pp}}^{\mathrm{G}}$ values $(\leqslant 5 \mathrm{G})$ is quite small —only about $20 \%$ - and this is well within the uncertainty of the $N_{s}$ values. However, for $N_{s}$ values around $10^{19} \mathrm{~cm}^{-3}$ an approximate doubling is required. Of course the values of $N_{s}$ given in Table I are average values for the film whereas the dipolar broadening is determined by the local values. It may be that as the transition from DLC to PLC occurs with the rise in hydrogen content that the spin concentration is not uniformly reduced so that some local values would exceed the average value. This is plausible since we have seen that the unpaired spins are likely to be associated with $\pi$-type radicals but when the bias voltage is reduced below about $100 \mathrm{~V}$, the protonated, fourfold coordinated, $\mathrm{C}$ bonding configurations increase more rapidly that the protonated, threefold coordinated ones. ${ }^{36}$ However, for $N_{s} \leqslant 10^{18} \mathrm{~cm}^{-3}$, the $N_{s}$ value will have little effect on the overall linewidth and $\Delta B_{\mathrm{pp}}^{\mathrm{G}}$ values since the dipolar contribution is already so small.

In summary, we propose that for $V_{b}<100 \mathrm{~V}$ the linewidth and shape are dominated by two contributions-the dipolar interaction which gives a line shape close to Lorentzian and a Gaussian contribution arising from unresolved $\mathrm{H}^{1}$ hyperfine lines. As $V_{b}$ is decreased and the hydrogen content increases while $N_{s}$ decreases, then the former contribution decreases and the latter increases. Exchange plays no part and this is confirmed by the fact that, as Table I shows, as $V_{b}$ is 
decreased below $100 \mathrm{~V}$ the optical gap continues to increase even though now the linewidth passes through a maximum and starts to decrease.

Before concluding we discuss two final matters. First, is it a good approximation to ignore the unresolved $\mathrm{H}^{1}$ hyperfine contribution to the linewidth and shape for $V_{b} \geqslant 100 \mathrm{~V}$. At $V_{b}=100 \mathrm{~V}, N_{s}$ is typically $>10^{20} \mathrm{~cm}^{-3}$ and the hydrogen content typically $<40$ at. \% so Eqs. (9) and (13) imply that the dipolar contribution exceeds, maybe considerably in some cases, the hyperfine contribution. As $V_{b}$ is increased further, $N_{s}$ does not change much initially but the hydrogen content continues to decrease. Therefore the approximation is reasonably good.

Finally, we comment on the results of Hoinkis et al. ${ }^{14}$ who measured the effect of changing the hydrogen content on the EPR linewidth from $a-\mathrm{C}: \mathrm{H}$. They found that at about 24 at. $\% \mathrm{H} \Delta B_{\mathrm{pp}}$ (measured in vacuum) reached a minimum of $2.8 \mathrm{G}$ with $N_{s}=4.5 \times 10^{20} \mathrm{~cm}^{-3}$. As the hydrogen content was increased the linewidth started to increase again whereas $N_{s}$ continued to fall and the approximate values are $\Delta B_{\mathrm{pp}}$ $=8 \mathrm{G}, N_{s}=1.5 \times 10^{20} \mathrm{~cm}^{-3}$ at 36 at. $\% \mathrm{H}$ and $\Delta B_{\mathrm{pp}}=10 \mathrm{G}$, $N_{s}=0.5 \times 10^{20} \mathrm{~cm}^{-3}$ at 39 at. $\% \mathrm{H}$. They interpreted this increase in $\Delta B_{\mathrm{pp}}$, for $>24$ at. $\% \mathrm{H}$, as due to an increase in the unresolved hydrogen hyperfine contribution. Our interpretation is different. First, at 24 at. \% H, Eqs. (9) and (13) imply that the dipolar contribution $\Delta B_{\mathrm{pp}}^{\mathrm{L}}=37 \mathrm{G}$ and hyperfine contribution $\Delta B_{\mathrm{pp}}^{\mathrm{G}}=4.5 \pm 1.2 \mathrm{G}$, and Eq. (12) then gives $\Delta B_{\mathrm{pp}}^{T}$ $=37.2 \mathrm{G}$, we suggest that the measured $\Delta B_{\mathrm{pp}}^{T}=2.8 \mathrm{G}$ is much less than this because the $s p^{2}$ content is high enough for there to be exchange narrowing. At 36 at. $\% \mathrm{H}$ the same calculations give $\Delta B_{\mathrm{pp}}^{\mathrm{L}}=12.2 \mathrm{G}, \Delta B_{\mathrm{pp}}^{\mathrm{G}}=6.5 \pm 1.8 \mathrm{G}, \Delta B_{\mathrm{pp}}^{T}$ $=15 \mathrm{G}$, which is closer to but still exceeds the measured value $\Delta B_{\mathrm{pp}}^{T}=8 \mathrm{G}$. We suggest that there is still some exchange narrowing but much less than before because increasing the hydrogen content will tend to decrease the $s p^{2}$ content and hence decrease wave function overlap. At 39 at. $\%$ $\mathrm{H}$ we calculate $\Delta B_{\mathrm{pp}}^{\mathrm{L}}=4 \mathrm{G}, \quad \Delta B_{\mathrm{pp}}^{\mathrm{G}}=7 \pm 2 \mathrm{G}, \quad \Delta B_{\mathrm{pp}}^{T}=9$ $\pm 2 \mathrm{G}$, which agrees with the measured value of $10 \mathrm{G}$. Thus we suggest that in the range $25-36$ at. $\% \mathrm{H}$ the dominant contributions to the width and shape are the dipolar and exchange interactions which will give a Lorentzian-like line as observed; these contributions change in this range because of changes to the $s p^{2}$ content and $N_{s}$. Only at 39 at. $\% \mathrm{H}$ (and above) does the hyperfine interaction make the dominant contribution and it together with the dipolar contribution then fully account for the linewidth; however, we predict that this spectrum has a Voigt shape. The effects they observe ${ }^{14}$ on decreasing from 39 to 24 at. $\% \mathrm{H}$ are therefore very similar to that of increasing $V_{b}$, in our case, from about 50 to $540 \mathrm{~V}$.

Hoinkis et al. ${ }^{14}$ also find that decreasing the hydrogen content below 24 at. $\% \mathrm{H}$ leads to an increase in width and, at 6 at. $\% \mathrm{H}, \Delta B_{\mathrm{pp}}$ has increased to about $88 \mathrm{G}$. At the same time the resistivity has fallen to just below $10 \Omega \mathrm{cm}$ and they reasonably suggest that this increase in width is due to a reduction in the spin relaxation times due to the interaction of the unpaired spins with the conduction electrons. This is not a situation which arose in our samples, although it is likely to account for the increase in linewidth which has been reported $^{6,7,41,42}$ for $a-\mathrm{C}: \mathrm{H}$ annealed to sufficiently high temperatures.

\section{CONCLUSIONS}

We have provided a comprehensive interpretation of the way in which the EPR linewidth and shape change as the structure of the $a-\mathrm{C}: \mathrm{H}$ is changed from polymerlike to diamondlike to graphitelike by increasing the negative self-bias voltage from about 10 up to $540 \mathrm{~V}$. The change from PLC to DLC in the range 10 to about $50-100 \mathrm{~V}$ is accompanied by an increase in linewidth, a line shape change, and a large increase in unpaired spin concentration. The linewidth increases because the increase in the dipolar contribution more than compensates for the decrease in the contribution from unresolved hydrogen hyperfine splittings. The former contribution is close to Lorentzian and the latter Gaussian, and the overall line shape should have a Voigt profile, as we observe at $V_{b}=50 \mathrm{~V}$, but gradually becomes Lorentzian as $V_{b}$ is increased. At $V_{b}=100 \mathrm{~V}$ the linewidth has reduced, probably because the hyperfine contribution has fallen to negligible proportions, and can be accounted for solely in terms of the dipolar interaction. Thus for $V_{b} \leqslant 100 \mathrm{~V}$ the exchange interaction, if present, has a negligible effect on the linewidth and this is borne out by the fact that, as $V_{b}$ is decreased below 50 $\mathrm{V}$, the optical gap continues to increase whereas the linewidth decreases. The hydrogen content is related empirically to the Gaussian, hydrogen hyperfine, contribution by $\Delta B_{\mathrm{pp}}^{\mathrm{G}}(\mathrm{G})=(0.18 \pm 0.05) \times($ at. $\% \mathrm{H})$.

As $V_{b}$ is increased above $100 \mathrm{~V}$ the linewidth decreases below that expected from the dipolar contribution alone, and this is attributed to exchange narrowing which becomes more pronounced as $V_{b}$ is increased. The increase in exchange interaction is attributed to the increased wave function overlap arising from greater unpaired spin delocalization which in turn arises from the increased $s p^{2}$ content. In this regime the line shape is Lorentzian and the optical gap is correlated with the linewidth. At the maximum $V_{b}$ of $540 \mathrm{~V}$ the exchange frequency, $\omega_{e}$, is estimated to be $1.2 \times 10^{10} \mathrm{rad} \mathrm{s}^{-1}$ and there is also some evidence for spin clustering.

Evidence for the existence of exchange is that the spinlattice relaxation time decreases and the spin-spin relaxation time increases as $V_{b}$ is increased in a way similar to that observed for DPPH as its concentration is increased. For all samples investigated the characteristics of the EPR signal are consistent with it being associated with $\pi$-type rather than $\sigma$-type radicals.

\section{ACKNOWLEDGMENTS}

This work was supported by a Basic Research Grant (No. SC/1998/704) from Enterprise Ireland. The authors thank Dr. D. Carey for the preparation of the PLC sample at $V_{b}$ $=10 \mathrm{~V}$. 
${ }^{1}$ J. Robertson, Adv. Phys. 35, 317 (1986); Philos. Mag. B 76, 335 (1997).

${ }^{2}$ P. Koidl, Ch. Wild, B. Dischler, J. Wagner, and M. Ramsteiner, Mater. Sci. Forum 52, 41 (1989); J. Robertson and E. P. O’Reilly, Phys. Rev. B 35, 2946 (1987).

${ }^{3}$ A. Bubenzer, B. Dischler, G. Brandt, and P. Koidl, J. Appl. Phys. 54, 4590 (1983).

${ }^{4}$ J. Robertson, in Amorphous Carbon: State of the Art, edited by S. R. P. Silva, J. Robertson, W. I. Milne, and G. A. J. Amaratunga (World Scientific, Singapore, 1998) p. 32.

${ }^{5}$ J. Robertson, Phys. Rev. B 53, 16302 (1996).

${ }^{6}$ D. J. Miller and D. R. McKenzie, Thin Solid Films 108, 257 (1983).

${ }^{7}$ J. González-Hernández, R. Asomoza, and A. Reyes-Mena, Solid State Commun. 67, 1085 (1988).

${ }^{8}$ S. Schütte, S. Will, H. Mell, and W. Fuhs, Diamond Relat. Mater. 2, 1360 (1993).

${ }^{9}$ J. Ristein, J. Schäfer, and L. Ley, Diamond Relat. Mater. 4, 508 (1995).

${ }^{10}$ S. R. P. Silva, J. Robertson, G. Rusli, and G. A. J. Amaratunga, Philos. Mag. B 74, 369 (1996).

${ }^{11}$ R. Kleber, K. Jung, H. Ehrhardt, I. Mühling, K. Breuer, H. Metz, and F. Engelke, Thin Solid Films 205, 274 (1991).

${ }^{12}$ M. Chhowalla, J. Robertson, C. W. Chen, S. R. P. Silva, C. A. Davis, and G. A. J. Amaratunga, J. Appl. Phys. 81, 139 (1997).

${ }^{13}$ M. Weiler, S. Sattel, T. Giessen, K. Jung, H. Ehrhardt, V. S. Veerasamy, and J. Robertson, Phys. Rev. B 53, 1594 (1996).

${ }^{14}$ M. Hoinkis, E. D. Tober, R. L. White, and M. S. Crowder, Appl. Phys. Lett. 61, 2653 (1992).

${ }^{15}$ J. P. Goldsborough, M. Mandel, and G. E. Pake, Phys. Rev. Lett. 4, 13 (1960).

${ }^{16}$ F. Giorgis, A. Tagliaferro, and M. Fanciulli, in Amorphous Carbon: State of the Art, edited by S. R. P. Silva, J. Robertson, W. J. Milne, and G. A. J. Amaratunga (World Scientific, Singapore, 1998) p. 143.

${ }^{17}$ S. D. Berger, D. R. McKenzie, and P. J. Martin, Philos. Mag. Lett. 57, 285 (1988).

${ }^{18}$ S. R. P. Silva, R. U. A. Khan, A. P. Burden, J. V. Anguita, J. M. Shannon, B. J. Sealy, A. J. Papworth, C. J. Kiely, and G. A. J. Amaratunga, Thin Solid Films 332, 118 (1998).

${ }^{19}$ C. P. Poole, Electron Spin Resonance (Wiley, New York, 1983) p. 476.
${ }^{20}$ See Ref. 19, p. 489.

${ }^{21}$ G. Rusli, G. A. J. Amaratunga, and S. R. P. Silva, Opt. Mater. 6, 93 (1996).

${ }^{22}$ D. Arcon, P. Cevc, R. Blinc, I. Pocsik, M. Koos, Z. Trontelj, and Z. Jaglicic, in Molecular Nanostructures, edited by J. Fink, M. Mehring, and S. Roth (World Scientific, Singapore, 1998), p. 533.

${ }^{23}$ Q. Zhang, S. F. Yoon, Rusli, J. Ahn, H. Yang, and D. Bahr, J. Appl. Phys. 84, 5538 (1998).

${ }^{24}$ H. Seidel and H. C. Wolf, in Physics of Color Centers, edited by W. Fowler (Academic Press, New York, 1968), p. 557.

${ }^{25}$ S. Sattel, J. Robertson, H. Ehrhardt, J. Appl. Phys. 82, 4566 (1997).

${ }^{26}$ J. Robertson, J. Non-Cryst. Solids 198-200, 615 (1996).

${ }^{27}$ See Ref. 19, p. 590.

${ }^{28}$ A. Abragam and B. Bleaney, Electron Paramagnetic Resonance of Transition Ions (Clarendon Press, Oxford, 1970) p. 110.

${ }^{29}$ R. C. Pastor and R. H. Hoskins, J. Chem. Phys. 32, 264 (1960).

${ }^{30}$ C. P. Slichter, Principles of Magnetic Resonance (SpringerVerlag, Berlin, 1992).

${ }^{31}$ P. W. Anderson and P. R. Weiss, Rev. Mod. Phys. 25, 269 (1953).

${ }^{32}$ A. Abragam, Principles of Nuclear Magnetism (Clarendon Press, Oxford, 1996) p. 126.

${ }^{33}$ J. C. Dawson and C. J. Adkins, J. Phys.: Condens. Matter 7, 6297 (1995).

${ }^{34}$ J. P. Sullivan and T. A. Friedmann, Amorphous Carbon: State of the Art (World Scientific, Singapore, 1998), p. 281.

${ }^{35}$ A. Ilie, N. M. J. Conway, B. Kleinsorge, J. Robertson, and W. I. Milne, J. Appl. Phys. 84, 5575 (1998).

${ }^{36}$ M. A. Tamor, W. C. Vassell, and K. R. Carduner, Appl. Phys. Lett. 58, 592 (1991).

${ }^{37}$ A. M. Stoneham, J. Phys. D 5, 670 (1972).

${ }^{38}$ A. Sadki, Y. Bounouh, M. L. Theye, J. von Bardeleben, J. Cernogora, and J. L. Fave, Diamond Relat. Mater. 5, 439 (1996).

${ }^{39}$ B. R. Weinberger, E. Ehrefreund, A. Pron, A. J. Heeger, and A. G. MacDiarmid, J. Chem. Phys. 72, 4749 (1980).

${ }^{40}$ A. Carrington and A. D. McLachlan, Introduction to Magnetic Resonance (Harper and Row, New York, 1967), pp. 77, 83, 90.

${ }^{41}$ S. Orzeszko, W. Bala, K. Fabisiak, and F. Rozploch, Phys. Status Solidi A 81, 579 (1984).

${ }^{42}$ R. C. Barklie, M. Collins, J. Cunniffe, and S. R. P. Silva, Diamond Relat. Mater. 7, 864 (1998). 